\title{
Röntgenfeinstruktur-Untersuchungen an Fischschuppen ${ }^{1}$ )
}

\section{Von UlRICH A. CoRTI und WILLFRIED EPPRECHT}

(Mitteilung aus der Eidg. Anstalt für Wasserversorgung, Abwasserreinigung und Gewässerschutz an der Eidg. Technischen Hochschule, Zürich, und dem Röntgenlaboratorium der Eidg. Materialprüfungs- und Versuchsanstalt für Industrie, Bauwesen und Gewerbe, Zürich)

Eingegangen am 8. Februar 1950

Zu den Problemen, die sich beim Versuch einer Totalanalyse der am Aufbau des Fischkörpers beteiligten Komponenten stellen, gehört nicht zuletzt auch die Untersuchung der Scbuppensubstanz. In der 4. Abhandlung $[4]^{2}$ ) der vorliegenden Berichtsreihe haben wir den Gesamtstickstoffgehalt und die Ausbeute an Asche der Schuppen diverser Arten von Süßwasserfischen mitgeteilt. Die Ausbeute an Asche variierte bei den geprüften Schuppen (lufttrocken) zwischen $27,6 \%$ (Schleie) und $58,9 \%$ (Flußbarsch).

Analysen der anorganischen Komponente von Fischschuppen sind schon mehrfach vorgenommen worden. So gibt z. B. W. Wunder [I3] (nach Cheuvreul) nachstehende Werte an:

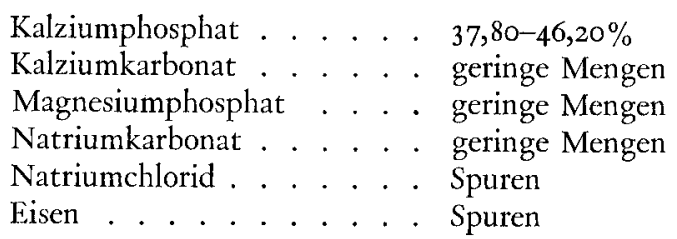

1) 7. Mitteilung siehe diese Zeitschrift, S. 94 .

2) Die Ziffern in eckigen. Klammern verweisen auf das Literaturverzeichnis auf S. 103/104. 
H. WEISKE [I2] ermittelte in Fischschuppen folgende Daten:

\begin{tabular}{|c|c|c|c|}
\hline & & Cyprinus carpio & Esoxlucius \\
\hline Kalk $(\mathrm{CaO})$. & & I $5,98 \%$ & $21,93 \%$ \\
\hline Magnesium $(\mathrm{MgO})$ & & $0,48 \%$ & $0,51 \%$ \\
\hline Phosphorsäure $\left(\mathrm{P}_{2} \mathrm{O}_{5}\right)$ & $\cdot$ & . $\quad \mathrm{3}, \mathrm{I} 2 \%$ & I $8,00 \%$ \\
\hline Kohlensäure $\left(\mathrm{CO}_{2}\right)$. & & $\mathrm{I}, 43 \%$ & $2,30 \%$ \\
\hline
\end{tabular}

Auf Grund dieser Feststellungen resultiert, daß die Mineralsubstanz der Fischschuppen zur Hauptsache aus Kalziumphosphat besteht.

Die an Knochen durchgeführten Untersuchungen $[2,3,5,6,9]$ legten u. a. die Frage nach der Zuordnung des in den Fischschuppen vorhandenen Kalziumphosphates zu einer bestimmten Kristallart nahe.

Zur Abklärung dieser Frage wurden saubere Schuppen folgender Fischarten röntgenologisch zunächst vermittels Debye-Scherrer-Aufnahmen untersucht:

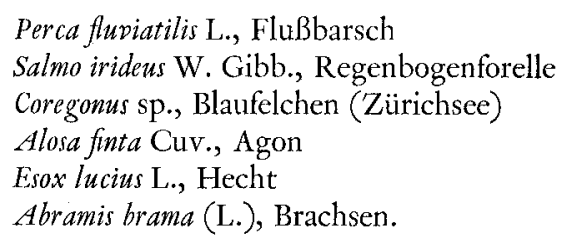

Die Röntgenaufnahmen erfolgten in einer Pulver-Kamera (Durchmesser II $\mathrm{mm}$ ) mit Cu-K-Strahlung und auf den Film aufgelegter Al-Folie. Die Belichtungszeit betrug $2 \frac{1}{2}$ Stunden. Aus den Schuppen von Salmo irideus und Abramis brama wurde je ein feines Stäbchen herausgeschnitten und diese Präparate der Röntgenanalyse unterworfen. Es ergaben sich dabei Diagramme, wie eines beispielsweise in Abb. I (unbehandelte Brachsenschuppe) wiedergegeben ist.

Diese Röntgendiagramme lassen eindeutig darauf schließen, daß in den geprüften Schuppen Apatit, und zwar vermutlich Hydroxylapatit, $\mathrm{Ca}_{5}\left(\mathrm{PO}_{4}\right)_{3}$ $(\mathrm{OH})$, in kristallisierter Form vorhanden ist. Aus der Intensität und Breite der Interferenzlinien läßt sich ableiten, daß die Einzelkristalle sehr geringe lineare Abmessungen haben. Die durchschnittliche Kristallgröße beträgt mutmaßlich kaum $\mathrm{ro}^{-6} \mathrm{~cm}$.

Da die Interferenzlinien, welche am unbehandelten Material gewonnen wurden, sehr breit sind, läßt sich die Röntgenanalyse dieser Schuppen nur schwer durchführen. Es wurden daher ähnliche Verfahren angewandt, wie sie bei Silikoseuntersuchungen üblich sind. Die Schuppen wurden zunächst bei steigenden Temperaturen sorgfältig entgast und dann während einer Stunde bei $600^{\circ} \mathrm{C}$ geglüht. Bei der Veraschung von Organismenteilen hat 
es sich gezeigt, daß diese Operation keine zersetzende Wirkung auf den Apatit ausübt; es tritt nur eine Vergröberung der Apatitkristalle auf, die für die weitere Analyse gerade wünschenswert ist. Die so veraschten Schuppen wurden nun der Röntgenanalyse unterworfen, und die auf diese Weise erhaltenen Filme entsprechen den Abb. 3 (Alosa finta) und 4 (Salmo irideus). Man erkennt in ihnen eine Folge von vielen, zum Teil zienlich scharfen Interferenzlinien, welche sich einwandfrei bekannten Kristallarten zuordnen lassen.

Abb. 2 zeigt zum Vergleich das Diagramm eines aus einer verknöcherten Vene stammenden Hydroxylapatites, und es fällt sofort auf, daß dieses

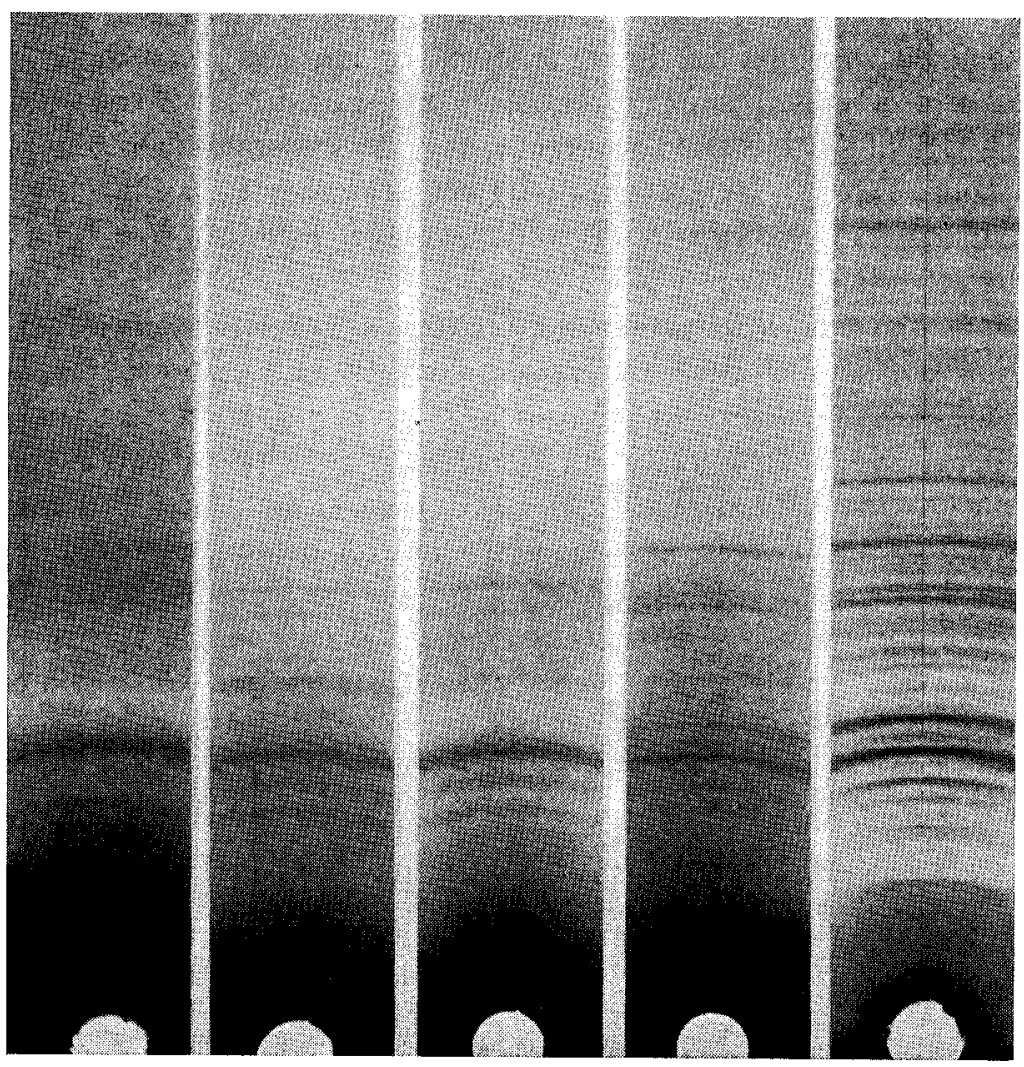

1. Abb.: unbehandelte Brachsenschuppe.

2. Abb.: verknöcherte menschliche Vene (Hydroxylapatit).

3. Abb.: Agonschuppe bei $600^{\circ} \mathrm{C}$ geglüht.

4. Abb.: Regenbogenforellenschuppe bei $600^{\circ} \mathrm{C}$ geglüht.

5. Abb.: $\beta-\mathrm{Ca}_{8}\left(\mathrm{PO}_{4}\right)_{2}$, künstlich. 
Interferenzmuster mit demjenigen des geglühten Agonpräparates übereinstimmt. Andererseits stellt Abb. 5 das an $\beta-\mathrm{Ca}_{3}\left(\mathrm{PO}_{4}\right)_{2}$ hergestellte Röntgendiagramm dar, das seinerseits weitgehend mit demjenigen der veraschten Schuppe von Salmo irideus übereinstimmt.

Durch derartige Vergleiche wurde folgendes festgestellt:

a) Die Schuppen von Brachsen und Regenbogenforelle enthalten im Originalzustand in röntgenographisch nachweisbarer Menge und Form nur Hydroxylapatit;

b) nach Glihen bei $600^{\circ} \mathrm{C}$ weisen die Schuppen von Felchen, Agon, Hecht, Flußbarsch die Kristallart Hydroxylapatit auf, hier nun in etwas grobkörnigerer Form als im Originalzustand. Demgegenüber weisen die Diagramme der veraschten Schuppen des Brachsen und der Regenbogenforelle ein Interferenzsystem auf, das demjenigen von $\beta$ - $\mathrm{Ca}_{3}\left(\mathrm{PO}_{4}\right)_{2}$ deutlich nähersteht als demjenigen des Hydroxylapatites.

Diese Resultate müssen folgendermaßen gedeutet werden: Die Schuppen von Agon, Felchen, Flußbarsch und Hecht bestehen u. a. eindeutig aus kristallinem Hydroxylapatit, der durch Glühen lediglich eine Kornvergröberung erfährt.

Die Schuppen des Brachsen und der Regenbogenforelle enthalten im Originalzustand ebenfalls Hydroxylapatit in kristalliner Form; beim Gliihen auf $600^{\circ} \mathrm{C}$ verhalten sie sich jedoch etwas anders als die vorgenannten Schuppen. Das Glühen erzeugt in ihnen kristallines Trikalziumphosphat, das im Originalzustand der Schuppen nicht in kristalliner Form vorlag. Nach TRÖMEL und MöLlER [II] kann diese Erscheinung folgendermaßen erklärt werden: aus wäßriger Lösung ausgefällter Hydroxylapatit kann mehr oder weniger $\mathrm{PO}_{4}$ adsorbieren; beim Glühen entsteht im Falle von größeren $\mathrm{PO}_{4}$-Adsorptionsmengen durch Reaktion mit Hydroxylapatit Trikalziumphosphat.

Das Röntgendiagramm der geglühten Schuppen von Brachsen und Regenbogenforelle läßt infolge der Ähnlichkeit der Diagramme von Apatit und Trikalziumphosphat nicht mit Sicherheit beurteilen, ob auch im geglühten Präparat noch Apatit vorhanden ist; immerhin scheint dies sehr wahrscheinlich.

Beim reinen Hydroxylapatit ist das Verhältnis P:Ca wie $\mathrm{I}: 2, \mathrm{I} 5$, beim Trikalziumphosphat wie I I I,94. Aus den weiter oben angegebenen Analysenwerten betreffend die Schuppen des Karpfens und Hechtes errechnet sich das Verhältnis P:Ca mit I:I,99 (Karpfen) bzw. I : I,99 (Hecht).

Unter dem Polarisationsmikroskop zeigen die unbehandelten Fischschuppen eine deutliche optische Anisotropie, was vermuten ließ, daß die 
vorhandenen kristallisierten Bestandteile in geregelter Art und Weise in die organische Substanz eingelagert sind. Es wurde daher ein kleiner Teil einer Brachsenschuppe, der unter gekreuzten Nicols gleichmäßig auslöscht, mit einem Röntgenstrahl senkrecht bestrahlt und auf einem planen Film die abgebeugten Interferenzen nach dem Laue-Verfahren aufgefangen. Es zeigte sich darnach, daß kontinuierliche Debye-Scherrer-Ringe entstehen, woraus zu schließen ist, daß die Apatitkriställchen im durchstrahlten Bereich keine merkliche Regelung besitzen.

Die Anisotropieeffekte der Schuppen, welche im Polarisationsmikroskop gefunden werden, werden offenbar durch andere Ursachen hervorgerufen. Möglicherweise ist die organische Substanz in gewissem Sinne geregelt struiert. Vergleiche hierzu auch die Ausführungen von W. J. SCHMIDT [Io] und VALENTIN (zitiert bei W. WUNDER).

Es ist in diesem Zusammenhang noch darauf hinzuweisen, daß die in unserer früheren Abhandlung [4] mitgeteilten Gesamtstickstoffwerte als relativ sehr hoch zu taxieren sind. So berechnet sich z. B. der Gesamtstickstoffgehalt der lufttrockenen Schuppe des Blaufelchens (Zürichsee), bezogen auf den Glühverlust ( $\sim$ «organische Substanz») zu ca. 19,5\% und bei der Schuppe des Hechtes zu ca. $17,4 \%$.

\section{SUMMARY}

We have found that most of the inorganic components in the scales of different inland fish species consist of crystallized hydroxyl apatit (part of which contains adsorbed $\left[\mathrm{PO}_{4}\right]$ ), in analogy to the mineral constituents of the bones of other vertebrate animals.

\section{LITERATURVERZEICHNIS}

[1] BETHE, A., Über die Silbersubstanz in der Haut pon Alburmus lucidus, Z. phys. Chem. $20,472-477$ (1895).

[2] BRANDENBerger, E., und SCHINZ, H. R., Die Natur der organischen Knochensubstanz, Experientia 4, 59-60 (1948).

[3] BRANDenberger, E., und Schinz, H. R., Zielsetzung und Ergebnisse systematiscber Feinstrukturuntersucbungen mittels der Röntgeninterferenzen beim Menschen, Bull. Schweiz. Akad. Med. Wiss. 3, 262-268 (I948).

[4] CoRTI, U. A., Über den Gebalt bzm. die Ausbeute perschiedener Fischorgane an Trockensubstanz, Gesamtstickstoff und Ascbe, Schweiz. Z. Hydrol. 11, 323-33I (1949). 
[5] Dallemagne, M. J., und Brasseur, H., La nature du sel principal de l'os étudiée par la diffraction des rayons $X$, Experientia 3, 469 (1947).

[6] Dallemagne, M. J., The Theory of primary calcification in Bone, Nature 161, II 5 (1948).

[7] HASE, A., Über das Schuppenkleid der Teleostier, Jenaische Z. Naturwiss. 42, 607-668 (I907).

[8] Herzog, R. O., und Gonell, H. W., Utber Kollagen, Ber. dtsch. chem. Ges. 58, 2228-2230 (1925).

[9] Niggli, P., und BrandenbergLR, E., Die Bedeutung röntgenographiscber Kristallstrukturuntersucbungen für die Medizin, Acta Radiologica 15, 350-363 (I934).

[ı] SснмiDT, W. J., Die Bausteine des Tierkörpers im polarisierten Licbt (Bonn I924).

[II] Trömel, G., und Möller, H., Die Bildung schwer löslicher Calciumphosphate aus wäßriger Lösung und die Bezielsungen dieser Phosphate zur Apatitgruppe, $\mathrm{Z}$. anorg. allg. Chemie 206, 227-240 (I932).

[12] Wisske, H., Über die Zusammensetzung pon Fiscbscbuppen und Fischknocben, Z. phys. Chem. 7, 466-473 (1 882/83).

[13] Wunder, W., Pbysiologie der Süfspasserfische Mitteleuropas, in: DEMOLL, R., und MaIER, H. N., Handbucb der Binnenfiscberei Mitteleuropas, Bd. II B (Stuttgart I936). 